\title{
Electrical stimulation to reduce the overload in upper limbs during sitting pivot transfer in paraplegic: a preliminary study
}

\author{
Ana Claudia G. Lopes (1,2), Claudia Ochoa-Diaz (3), Roberto S. Baptista (3), Lucas \\ O. Fonseca (3), Charles Fattal (4), Christine Azevedo Coste (5), Antônio P. L. Bó (3), \\ Emerson Fachin-Martins (2) \\ (1) SARAH Network Rehabilitation Hospitals, Brasília, Brazil; (2) NTAAI, Faculdade de \\ Ceilândia, Universidade de Brasília, Brasília, Brazi; (3) LARA, Faculdade de Tecnologia, \\ Universidade de Brasília, Brasília, Brazil; (4) Centre de Réhabilitation CRF, COS DIVIO, \\ Dijon, France; (5) INRIA, LIRMM, Montpellier, France
}

This article is distributed under the terms of the Creative Commons Attribution Noncommercial License (CC BY-NC 4.0) which permits any noncommercial use, distribution, and reproduction in any medium, provided the original author(s) and source are credited.

\begin{abstract}
Transfer is a key ability and allows greater interact with the environment and social participation. Conversely, paraplegics have great risk of pain and injury in the upper limbs due to joint overloads during activities of daily living, like transfer. The main goal of this study is to verify if the use of functional electrical stimulation (FES) in the lower limbs of paraplegic individuals can assist the sitting pivot transfer (SPT). The secondary objective is to verify if there is a greater participation of the lower limbs during lift pivot phase. A preliminary study was done with one complete paraplegic individual. Temporal parameters were calculated and a kinetic assessment was done during the SPT. The preliminary results showed the feasibility of FES for assisting the SPT.

Key Words: spinal cord injuries, paraplegia, functional electrical stimulation, kinetics, biomechanics, rehabilitation
\end{abstract}

Eur J Transl Myol 26 (4): 278-282

Transfer is a key ability to gain mobility, independence, allows greater interaction with environment, social participation and improves quality of life for individuals with spinal cord injury (SCI). ${ }^{1-4}$ The most performed type of transfer by paraplegic subjects is sitting pivot transfer (SPT).$^{5,6}$ In most of the cases, they have potential to perform this task independently between 15 to 20 times a day on average. ${ }^{5}$ This large number of transfers also contributes to the development or perpetuation of secondary upper limbs (UL) musculoskeletal impairments over time and it is know that after SCI, individuals have great risks of pain and injury in the UL due to joint overloads during activities of daily living. ${ }^{5-8}$

Neuromuscular electrical stimulation (NMES) can be used as a potential technological resource to assist these people during transfers. NMES used as a functional way, is known as functional electrical stimulation (FES). Murphy et al. in 2014 demonstrated that FES applied on extensor muscles of the trunk favors the balance during the transfer. ${ }^{9}$ Jovic et al. suggested in a previous study in 2013 with healthy subjects that FES on lower limbs (LL) may decrease the overload on UL during the SPT in paraplegics, ${ }^{10}$ and, two years later, Jovic et al. showed that FES on LL decreases the overload on UL during the transfer from sitting to standing in individuals with low quadriplegia and paraplegia. ${ }^{11}$ So far, we did not find reports of FES use during the SPT on SCI individuals. Therefore, any information about the effect of FES in this population would be a valuable contribution to think about new strategies to aid SCI subjects during SPTs.

\section{Material and Methods}

\section{Participant}

The participant of this study is a male subject (37 years old, $1.70 \mathrm{~m}, 67.40 \mathrm{~kg}$ and manual preference for right hand) with complete sensory-motor thoracic traumatic spinal cord injury (T9), AIS A (American Spinal Injury Association Impairment Scale) and with 3 years postSCI. He is a manual wheelchair user who is capable to perform a SPT without physical or technical assistance, routinely using this type of transfer in daily life for both sides (right/left). Additionally, he does not have any condition that affects the ability to transfer or the use of electrical stimulation. 


\section{Electrical stimulation to reduce the overload in upper limbs on SPT}

Eur J Transl Myol 26 (4): 278-282

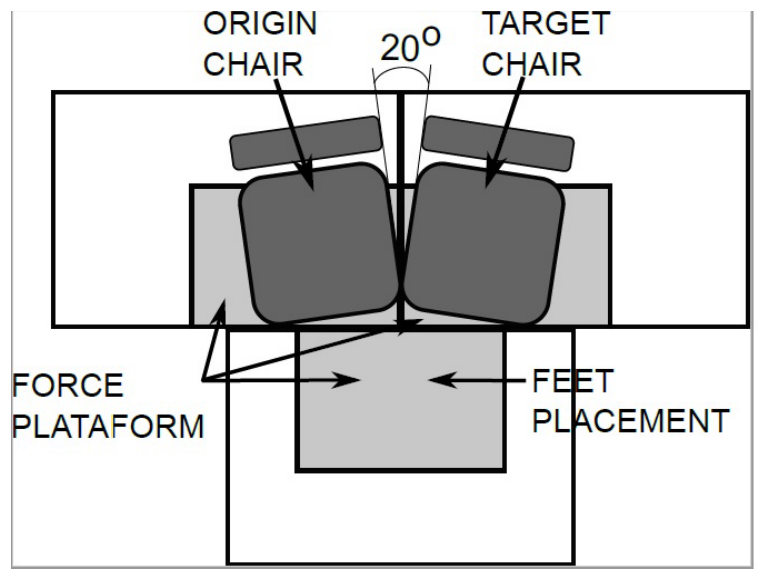

Fig 1. Experimental set up.

\section{Experimental Set Up}

The SPT were performed by the participant using two seats with the same characteristics $(42 \mathrm{~cm} \times 42 \mathrm{~cm})$ and with an angle of $20^{\circ}$ between them. The height of both seats $(48 \mathrm{~cm})$ resembles that of the wheelchair used by the participant. Beneath each seat, a force plat (Bertec Corporation; Columbus, Ohio) was installed in order to record reaction forces during the transfer. Additionally, another force plat was placed beneath the participant's feet, so the force caused by the stimulation could be also recorded. The seats were firmly attached to a wooden case placed between the seats and the force plates. Figure 1 presents a schematic of the experimental setup. The additional weight imposed by the seats and the wooden structure was compensated by software before running the experiments. Kinetic data were recorded with a sampling frequency of $200 \mathrm{~Hz}$. The bilateral femoral quadriceps (rectus femoral) of the participant were electrically stimulated (Hasomed, RehaStim) by surface electrodes with the following parameters: frequency of $50 \mathrm{~Hz}$ and pulse width of $450 \mu \mathrm{s}$, while the intensity varied according to the desired muscle contraction grades from the Manual Muscle Test (MMT). The intensity levels were identified while the individual was sitting on his wheelchair: $38 \mathrm{~mA}$ to reach grade 3 in MMT; 48mA to reach grade 4- in MMT; $52 \mathrm{~mA}$ to reach grade 4 in MMT. The parameters were tested in the individual before running the SPT experiments. The start and stop of FES was done by verbal command of individual and it was manually controlled. The participant was encouraged to use his usual transfer strategy during the experiments. After a familiarization period, transfer trials were recorded on both directions.

\section{Data Analysis}

Figure 2 shows the force data of the three force plates during the SPT for both scenarios: with and without FES. Force plates 1 and 3 correspond to the ones placed beneath each seat, while force plate 2 recorded the feet

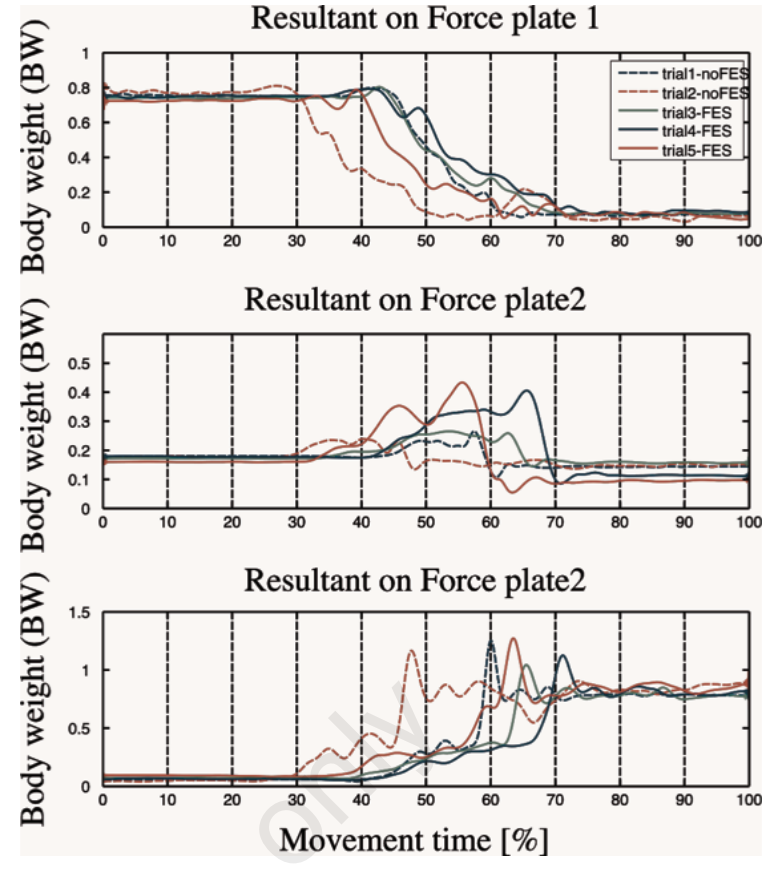

Fig 2. Resultant forces for each trial on both scenarios.

reaction forces. The force data was normalized to the participant body weight and the time was expressed as a percentage of the total movement time, corresponds to the total trial duration. The participant performed a total of eight trials with FES and four trials without FES. The trials chosen for analysis correspond to the ones in which the individual had the best performance in the SPT, based on some conditions verified through video documentation: transfer performed on the same side, hand maintenance in the same position along the transfer and a single lift movement with respect to the pelvis motion. According to the analysis of the video recorded for each trial, the best performance of the SPT was when the participant transferred from the left to the right side, therefore three trials with FES and two without FES were selected for further analysis. In this study the SPT division is the same as the one used in previous works. ${ }^{6,8}$ Hence, the pivot transfer is defined by the occurrence of three phases: pre-lift, lift-pivot and post-lift. For this preliminary study, only the force information was used to delimit the beginning and the end of each phase. The beginning of the pre-lift and the end of the post-lift, which delimit the overall transfer motion were determined by the start and the end of motion dynamics looking at the forces provided by the force plates 1 and 3, while the lift pivot resultant takes place when the resultant force of plate 1 starts to decrease (after attaining a certain threshold) and ends when the resultant from the force plate 3 reaches its maximum value. Figure 3 shows the transfer motion selected by each trial according to the lift-pivot phase detection explained above. In order to assess the effect 

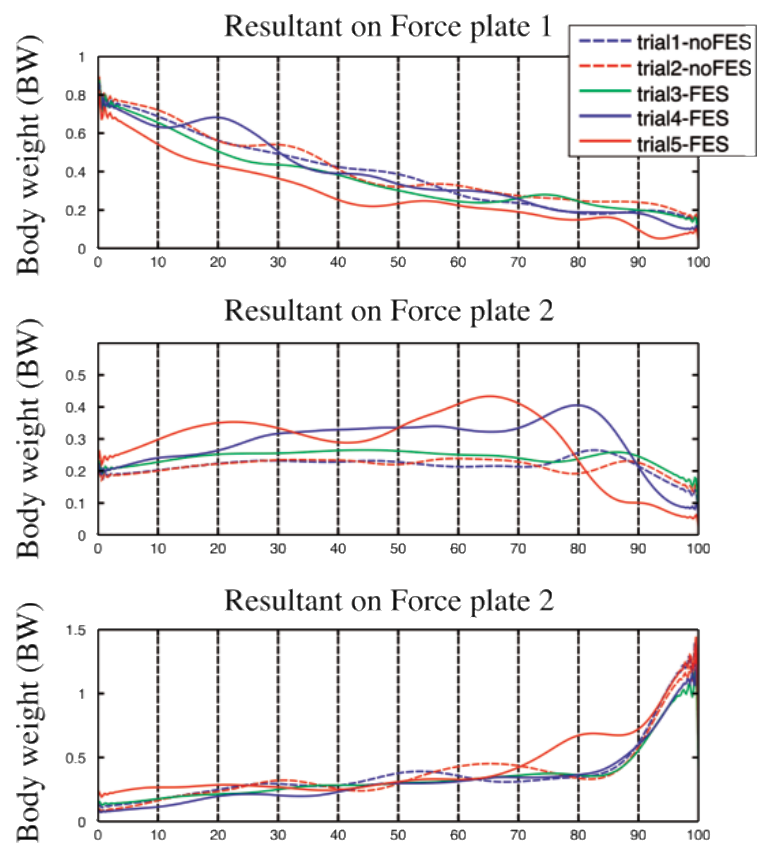

lift pivot face [\%]

Fig 3. Resultant forces recorded during the lift pivot phase in each trial.

of the FES during the SPT based on the force data from the three platforms, a calculation of the force impulse (the integral of force over time) for the resultant forces during the lift-pivot phase was performed. The idea of this calculation is to use this parameter to quantify the effect of FES on the LL. If the FES is capable to provoke a force on the LL, then the total force during the transfer will increase on the data recorded from force plate 2 when compared to the trials without FES. The force impulse of the rest of the plates should be the same or, in the best scenario, will decrease as a positive effect of the FES in aiding the subject to perform the SPT.

\section{Results}

The subject successfully executed the SPT in both scenarios. The total duration of SPT varies between 1.43 $\mathrm{s}$ to $2.45 \mathrm{~s}$, while the lift pivot phase in trials $1,2,3,4$, and 5 corresponds to $57 \%, 64 \%, 69 \%, 68 \%$ and $45 \%$ of the total SPT, respectively (see Table 1). The overall movement pattern was similar in all trials (Figure 2)

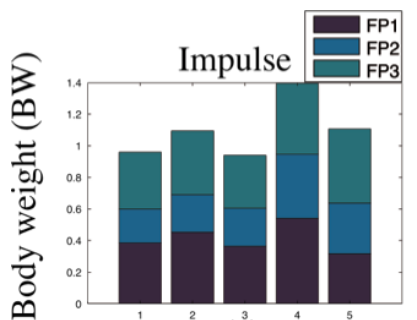

(a)

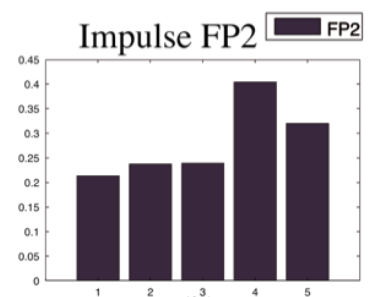

(b)

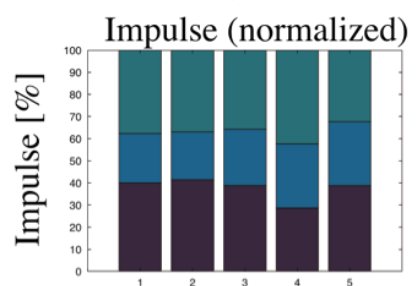

(c)
Trial

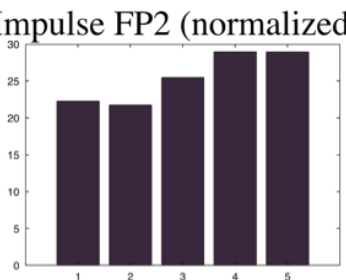

(d)
Fig 4. Impulse distribution on the three platforms: 4a. Impulse, 4b. Impulse FP2, 4c. Impulse (normalized), 4d. Impulse FP2 (normalized).

with the resultant force changing from plate 1 to 3 . A closer analysis of only the lift phase of the SPT (Figure 3) reveals some differences between the SPT with and without FES. Specifically, the force values in platform 2 are higher in trials 3, 4 and 5, compared to trials 1 and 2 . No differences in terms of force amplitude were observed on platforms 1 and 3 for all trials. Figure 4.a and 4.c show the results for the impulse in all platforms, as a stacked bar chart, in body weight units and normalized by the total amount of impulse in all the platforms. The calculation of impulse just for force plate 2 (LL forces) is also displayed on Figures 4.b and 4.d.

\section{Discussion}

There are indications that FES in LL assists SPT. Up to our knowledge, there is no study in the literature that uses the FES to assist the SPT, so it is not possible to compare our data with any other previous study. The total time of SPT are similar to that shown by Gagnon et al. ${ }^{6}$ ranging on average from 1.65 to 2.6 s, so FES did not modify the total transfer time, demonstrating that can

TABLE 1. Total time during the sitting pivot transfer (SPT) and the lift phase. The lift phase time is also expressed as percentage of the total during the transfer.

$\begin{array}{lllllllllll}\text { Time (s) } & \text { trial 1 } & \% & \text { trial 2 } & \% & \text { trial 3 } & \% & \text { trial 4 } & \% & \text { trial 5 } & \% \\ \text { SPT time } & 1.71 & 100 & 1.73 & 100 & 1.43 & 100 & 1.97 & 100 & 2.45 & 100 \\ \text { Lift phase } & 0.98 & 57.14 & 1.1 & 63.58 & 0.98 & 68.88 & 1.34 & 68.27 & 1.1 & 44.81\end{array}$


be a good resource that does not affect the agility during the paraplegic transfer. This is important to be considered, because if the assistive technology device in potential increases the total transfer time, it will not be practical on daily activities and the individual may have some resistance to use it. Gagnon et al. found that the lift pivot phase has an average duration of $40 \%$ of the total SPT cycle. ${ }^{6}$ In our experiments, the closest value was found in the FES trial with the highest intensity (trial 5). It seems that a higher stimulation intensity reduces the lift phase duration, which in turn may reduce UL overload. In more a detailed study of the lift pivot phase it is observed that the higher the current intensity of FES, the higher the muscular strength and greater the impulse on plate 2. This may indicate that the degree 4- in MMT of quadriceps muscle with FES is a good intensity to assist SPT. This threshold will be better studied when the research sample is enlarged. Additionally, the use of FES did not hinder the SPT movement and there were no clear negative effect, according to our preliminary analysis. The kinetics curves in Figure 3 indicate an increase in forces applied by the LL in the SPT during the trials with FES activation, which means that the muscular activity induced by the FES was converted into forces that may have a positive effect during the SPT favoring the movement. During the lift-pivot phase the subject's body weight is supported by the UL and LL. Therefore, the increase of the participation of the LL in the total impulse during the lift-pivot phase, as displayed in Figure 4 is an indication of alleviated effort in the UL. The feet support is almost $30 \%$ of the body weight. We need to investigate more if the increase of FES will produce a better participation of LL and a lower muscular demand of shoulder joint muscles, as well as a decrease impingement of the subacromial structures, which may minimize the risk of the joints during transfers. Physiotherapists and occupational therapists have little knowledge and clinical resources to assess SPT in clinical practice. There are few studies of this ability and little progress has been made in this area of expertise over the past years. The sliding board is the unique low cost assistive technology resource used worldwide, but few progresses have been made on new technological possibilities in this area. FES can be a potential alternative since it can be portable, lightweight, personalized and easy to use with surface electrodes. This study shows some evidence that FES of quadriceps may favor participation of LL during SPT, but the hypothesis that FES in LL decreases overload on UL should be further investigated in future studies.

\section{Author's Contribution}

ACGL: recruitment of volunteers, experimental and clinical setup implementation, clinical data acquisition; CO-D: experimental setup implementation, data acquisition, processing of data; RSB: experimental setup implementation, data acquisition, processing of data; LOF: experimental and technical setup implementation; CF: study design, expertise in FES applied to SCI rehabilitation; CA-C: protocol and setup design expertise; APLB: coordination of the technical aspects of the study, technical expertise in stimulator programming; EF-M: protocol design, advisor and coordination of the project with rehabilitation expertise.

\section{Acknowledgment}

We thank to the financial support to CACAO by INRIA and FAPDF, CAPES and CNPq and to the participants who give us motivation to continue in this work.

\section{Conflict of Interest}

The authors declare no conflict of interests.

\section{Corresponding Author}

Emerson Fachin-Martins, NTAAI, Faculdade de Ceilândia, Universidade de Brasília, Brasília, Brazil.

E-mail: efmartins@unb.br

\section{E-mail of coAuthors}

Ana Claudia G. Lopes: anacglopes10@gmail.com

Claudia Ochoa-Diaz: clau8a@gmail.com

Roberto S. Baptista: baptista@ieee.org

Lucas Oliveira da Fonseca:

Charles Fattal: cfattal@cos-asso.org lucasfonseca27@gmail.com

Christine Azevedo-Coste: christine.azevedo@inria.fr; Antônio Padilha Lanari Bó: apastilha@gmail.com

\section{References}

1. Marsolais EB, Scheiner A, Miller PC, R. et al. Augmentation of transfers for a quadriplegic patient using an implanted FNS system. Case report. Paraplegia 1994;32:573-9.

2. Triolo R, Bieri C, Uhlir J, Kobetic R. Implanted FNS systems for assisted standing and transfers for individuals with cervical spinal cord injuries. Arch Phys Med Rehabil, 1996;77:1119-28.

3. Gagnon D, Nadeau S,. Noreau L, etal. Electromyographic patterns of upper extremity muscles during sitting pivot transfers performed by individuals with spinal cord injury. J Electromyogr Kinesiol 2009;19:509-20.

4. Kankipati P, Boninger ML, Gagnon D,et al. Upper limb joint kinetics of three sitting pivot wheelchair transfer techniques in individuals with spinal cord injury. J Spinal Cord Med 2015;38:485-97.

5. Consortium for Spinal Cord Medicine and $P$. Veterans. Preservation of upper limb function following spinal cord injury: a clinical practice guideline for health-care professionals. J Spinal Cord Med 2005;28:434-70.

6. Gagnon D, Koontz AM, Mulroy SJ, et al. Biomechanics of Sitting Pivot Transfers Among Individuals with a Spinal Cord Injury: A Review of the Current Knowledge. Top Spinal Cord Inj Rehabil 2009;15:33-58. 
7. Desroches G, Gagnon D, Nadeau S, Popovic M. Magnitude of forward trunk flexion influences upper limb muscular efforts and dynamic postural stability requirements during sitting pivot transfers in individuals with spinal cord injury. $\mathrm{J}$ Electromyogr Kinesiol 2013;23:1325-33.

8. Gagnon D., Nadeau S, Noreau L,et al. Trunk and upper extremity kinematics during sitting pivot transfers performed by individuals with spinal cord injury. Clin Biomech 2008;23:279-90.

9 Murphy JO, Audu ML, Lombardo LM, et al. Feasibility of closed-loop controller for righting seated posture after spinal cord injury. J Rehabil Res Dev 2014;51:747-60.

10. Jovic J, Lengagne S, Fraisse P, Azevedo-Coste C. Impact of functional electrical stimulation of lower limbs during sitting pivot transfer motion for paraplegic people. Int $\mathbf{J} \mathrm{Adv}$ Robot Syst 2013;10:1-8.

11. Jovic J, Coste-Azevedo C, Fraisse Pet al. Coordinating Upper and Lower Body During FESAssisted Transfers in Persons With Spinal Cord Injury in Order to Reduce Arm Support. Neuromodulation 2015;18:736-43. 